\title{
Tratamento Cirúrgico Conservador da Hidrossalpinge: Laparoscopia ou Microcirurgia?
}

\author{
Conservative Surgical Treatment of Hydrosalpinx: \\ Laparoscopy or Microsurgery?
}

\begin{abstract}
Sérgio Conti Ribeiro, Alessandra de Araujo Silva, Carlos Roberto Izzo Nelson da Cruz Santos, Vicente Mário Izzo, José Aristodemo Pinotti
\end{abstract}

\begin{abstract}
RESUMO
Objetivos: analisar as taxas de gravidez após realização de correção laparoscópica e microcirúrgica de hidrossalpinge.

Métodos: no periodo de julho de 1996 a maio de 1999, foram tratadas 39 pacientes com hidrossalpinge, segundo protocolo de pesquisa previamente aprovado. As pacientes foram distribuidas, por sorteio, em dois grupos, de acordo com o tipo de acesso cirúrgico a ser utilizado: salpingostomia laparoscópica ou por laparotomia. Para análise dos resultados, as pacientes foram estratificadas de acordo com o grau de lesão tubária e as taxas de gestação nos dois grupos foram anotadas durante um intervalo de 24 meses.

Resultados: as taxas de gravidez foram de 35,3 e 33,3\%, respectivamente, após laparoscopia e microcirurgia. Em relação à gravidade da lesão tubária, 66,7\% das pacientes com lesões leves e 21,7\% das pacientes com lesões moderadas obtiveram sucesso na concepção. As taxas cumulativas de gravidez em um e dois anos, respectivamente, foram de 25,0 e 34,4\%. Houve um caso de gestação ectópica, correspondendo a 9,1\% de todas as gestações.

Conclusões: pacientes com lesão tubária leve ou moderada podem ser tratadas inicialmente por cirurgia e o sucesso na concepção é inversamente proporcional ao grau de acometimento tubário.
\end{abstract}

PALAVRAS-CHAVE: Esterilidade. Hidrossalpinge. Laparoscopia. Microcirurgia.

\section{Introdução}

A alteração funcional das tubas uterinas é uma das mais freqüentes causas de infertilidade, presente em aproximadamente $25 \%$ dos casais inférteis. Entre as principais causas de lesão tubária destacam-se a doença inflamatória pélvica, a endometriose, a gestação ectópica e as aderências pélvicas secundárias a intervenções cirúrgicas abdominais ${ }^{1}$.

Nas últimas três décadas, técnicas microcirúrgicas têm sido utilizadas para o tratamento de tubas anatomicamente comprometidas,

Disciplina de Ginecologia do Hospital das Clínicas da Faculdade de Medicina da Universidade de São Paulo.

Correspondência:

Sérgio Conti Ribeiro

Rua Itambé, 289, apto 1207

01239-001 - São Paulo - SP

Tel. (11) 3846-4111

e-mail: sergioribeirogo@aol.com com taxas de gestação intra-uterina entre $30 \mathrm{e}$ $35 \%{ }^{2}$. A primeira etapa da cirurgia é a lise de aderências, restaurando-se a anatomia tubo-ovariana normal. O passo seguinte é criar um novo óstio com uma mucosa fimbrial ou ampolar evertida adequadamente ${ }^{3}$.

Com o aprimoramento das técnicas de fertilização in vitro (FIV), obtendo-se taxas de gestação de 25 a 50\%, coloca-se em dúvida se existe ainda espaço para correção cirúrgica das alterações tubárias responsáveis pela infertilidade de diversos casais.

Recentemente, a laparoscopia foi introduzida como abordagem minimamente invasiva para tratamento de lesões tubárias, com resultados promissores, porém ainda há poucos estudos prospectivos comparando as duas vias de acesso. O objetivo deste estudo é avaliar as taxas de gestação após o tratamento laparoscópico ou microcirúrgico de pacientes com hidrossalpinge. 


\section{Pacientes e Métodos}

De acordo com protocolo de pesquisa desenvolvido no Hospital das Clínicas da Faculdade de Medicina da Universidade de São Paulo no período de julho de 1996 a maio de 1999, foram acompanhadas prospectivamente 39 pacientes com hidrossalpinge agendadas inicialmente para tratamento cirúrgico. Todas as pacientes foram avaliadas por meio do exame clínico, FSH no $3^{\circ}$ dia do ciclo, ultra-sonografia, análise seminal do parceiro, teste pós-coital, progesterona no $19^{\circ}$ dia, biópsia endometrial e histerossalpingografia. Pacientes com fator masculino associado ou outra causa de infertilidade foram excluídas previamente do protocolo e encaminhadas ao programa de FIV. Obteve-se consentimento pós-informado de todas pacientes, de acordo com as normas da Comissão de Ética em Pesquisa da referida instituição.

As pacientes encaminhadas para salpingostomia foram divididas aleatoriamente em dois grupos, de acordo com a técnica proposta: Grupo 1, laparoscopia e Grupo 2, microcirurgia laparotômica. As características das pacientes em relação à idade média (31,6 e 31,1 anos) e ao tempo de este- rilidade (2,5 e 1,9 anos) eram semelhantes nos Grupos 1 e 2 (Tabela 1).

Tabela 1 - Características das pacientes em relação a idade e tempo de esterilidade.

\begin{tabular}{lccc}
\hline & $\mathbf{n}$ & Idade (anos) & $\begin{array}{c}\text { Tempo de } \\
\text { esterilidade (anos) }\end{array}$ \\
\hline Grupo 1 (Laparoscopia) & 21 & $31,6(22-35)$ & $2,5(1-4)$ \\
Grupo 2 (Microcirurgia) & 18 & $31,1(24-35)$ & $1,9(1-5)$ \\
Total & 39 & $31,4(22-35)$ & $2,2(1-5)$ \\
\hline
\end{tabular}

Realizou-se laparoscopia diagnóstica no início de todos os procedimentos e, de acordo com (i) o diâmetro ampolar distal, (ii) espessamento da parede tubária, (iii) existência de pregas mucosas no local da neostomia e (iv) extensão e tipo de aderências, as lesões tubárias foram graduadas em leve, moderada ou grave ${ }^{4}$. Sete pacientes com doença grave foram excluídas do protocolo e encaminhadas para FIV. Entre as pacientes consideradas adequadas para correção cirúrgica, quatro apresentavam lesão leve unilateral, 11 lesão moderada unilateral, cinco com lesões leves bilaterais e $12 \mathrm{com}$ lesões moderadas bilaterais (Tabela 2).

Tabela 2 - Avaliação intra-operatória das aderências pélvicas e hidrossalpinge.

\begin{tabular}{lcccccc}
\hline & \multicolumn{3}{c}{ Unilateral $(\%)$} & \multicolumn{3}{c}{ Bilateral (\%) } \\
& Leve & Moderada & Grave & Leve & Moderada & Grave \\
\hline Grupo 1 $(n=21)$ & $2(9,5)$ & $5(23,8)$ & $1(4,7)$ & $2(9,5)$ & $8(38,1)$ & $3(14,2)$ \\
Grupo 2 $(n=18)$ & $2(11,1)$ & $6(33,3)$ & $1(5,5)$ & $3(16,6)$ & $4(22,2)$ & $2(11,1)$ \\
Total & $4(20,6)$ & $11(57,1)$ & $2(10,3)$ & $5(26,1)$ & $12(60,3)$ & $5(25,4)$ \\
\hline
\end{tabular}

Grupo 1: laparoscopia; Grupo 2: microcirurgia

A técnica para salpingostomia laparoscópica foi executada com um trocarte umbilical de $10 \mathrm{~mm}$ e duas punções auxiliares de $5 \mathrm{~mm}$, além da inserção de cânula intra-uterina para manipulação e realização de cromotubagem. Após a identificação da área tubária lesada, realizava-se incisão cruciforme na porção mais distal da tuba com tesoura, seguida de delicada cauterização bipolar na base de cada um dos quatro folhetos, de tal forma que a luz tubária podia ser visualizada, permitindo então o extravasamento do azul de metileno injetado pela cânula transcervical. Em alguns casos, foram realizados pontos com Mononylon ${ }^{\circledR}$ 6-0 para fixar os folhetos recém-abertos, para evitar recidiva da oclusão.

No grupo 2 utilizou-se incisão de Pfannenstiel de 8 a $10 \mathrm{~cm}$ de extensão. Após identificação do local da lesão (ponto de fusão das pregas) com o auxílio da magnificação com lupa, abriu-se o mes- mo com tesoura de íris ou cautério de alta freqüência com ponta em agulha. A partir desta abertura, realizava-se incisão cruciforme em 4 segmentos com cautério bipolar ou tesoura de íris, com posterior eversão da mucosa tubária por tração dos segmentos ou, se necessário, com o auxílio do gancho oftálmico. A seguir, fixava-se a extremidade distal de cada segmento à mucosa tubárea com Mononylon ${ }^{\circledR}$ 6-0, mantendo assim o pavilhão tubáreo aberto e comprovando-se a seguir a permeabilidade da tuba por cromotubagem. Todas as pacientes operadas foram seguidas por um período de 24 meses.

\section{Resultados}

Os procedimentos foram realizados com sucesso nos dois grupos. As pacientes submetidas a 
salpingostomia laparoscópica receberam alta hospitalar após 24 horas e as operadas por microcirurgia após 60 horas.

Nos primeiros seis meses após a cirurgia, uma paciente do Grupo 1 (laparoscopia) e outra do Grupo 2 (microcirurgia) engravidaram, ambas com lesão unilateral leve. Após 12 meses, as taxas cumulativas de gestação para o Grupo 1 e Grupo 2 foram $29,4 \%$ (4/ 17) e 26,6\% (4/15), respectivamente. Houve mais três casos de gestação intra- uterina 18 meses após a cirurgia e, após 24 meses, a incidência total de gestações foi de $35,3 \%$ no grupo submetido à laparoscopia e 33,3\% naquele submetido a microcirurgia (Tabela 3).

Em relação à gravidade da lesão tubária, $66,7 \%$ das pacientes com lesões leves e $21,7 \%$ das pacientes com lesões moderadas obtiveram sucesso na concepção. Ocorreu um caso de gestação ectópica tubária, diagnosticada sete meses após a salpingostomia por laparotomia (Tabela 3).

Tabela 3 - Resultados de gestação após tratamento cirúrgico da hidrossalpinge.

\begin{tabular}{|c|c|c|c|c|c|c|c|c|c|}
\hline & \multirow[b]{2}{*}{ Tempo } & \multicolumn{6}{|c|}{ Incidência de gestações intra-uterinas } & \multicolumn{2}{|c|}{ Gestações ectópicas } \\
\hline & & (meses) & 6 meses & 12 meses & 18 meses & 24 meses & Total & & \\
\hline Grupo 1 & Leve & Unilateral (2) & 1 & 1 & - & - & 2 & & - \\
\hline \multirow{6}{*}{$\begin{array}{l}\text { Laparoscopia } \\
(\mathrm{n}=17)\end{array}$} & & Bilateral (2) & & & & & & 6 & \\
\hline & & & & & & & & $3 \%)$ & - \\
\hline & & & - & 1 & - & - & 1 & & \\
\hline & Moderada & Unilateral (5) & - & 1 & - & 1 & 2 & & - \\
\hline & & Bilateral (8) & & & & & & & \\
\hline & & & - & - & 1 & - & 1 & & - \\
\hline Grupo 2 & Leve & Unilateral (2) & 1 & & 1 & - & 2 & & - \\
\hline \multirow{6}{*}{$\begin{array}{l}\text { Microcirurgia } \\
(n=15)\end{array}$} & & Bilateral (3) & & & & & & 5 & \\
\hline & & & & & & & & $3,3 \%)$ & \\
\hline & & & - & 1 & - & - & 1 & & - \\
\hline & Moderada & Unilateral (6) & - & 1 & - & - & 1 & & 1 \\
\hline & & Bilateral (4) & & & & & & & \\
\hline & & & - & 1 & - & - & 1 & & - \\
\hline
\end{tabular}

\section{Discussão}

A salpingostomia foi realizada pela primeira vez em $1967^{5}$. Desde então, o fator tubário vem sendo tratado por meio de microcirurgia ${ }^{6}$. Às vezes, é difícil diferenciar a oclusão infundibular completa (com uma fímbria presente) de uma hidrossalpinge verdadeira: o procedimento cirúrgico na primeira é classificado como fimbrioplastia e no segundo como salpingostomia ${ }^{6}$. A fimbrioplastia cursa com taxas de gravidez de $31-61 \%^{2,6}$.

A primeira série de salpingostomias laparoscópicas demonstrou resultados de fertilidade comparáveis àqueles obtidos pela microcirur$\mathrm{gia}^{2}$. A taxa precoce de gravidez intra-uterina após salpingostomia laparoscópica foi de $44 \%$, com $90 \%$ de permeabilidade pós-operatória para pelo menos uma tuba uterina, e não houve gestações extrauterinas. Ao contrário, apenas $27 \%$ das pacientes alcançaram gestações viáveis após salpingostomia microcirúrgica ${ }^{7}$. Na presente casuística verificou- se taxa de gravidez de 35,3 e 33,3\% após salpingostomia laparoscópica e microcirúrgica, respectivamente. Estratificando-se os grupos de acordo com a gravidade da lesão, as taxas de gravidez foram de 66,7 e $21,7 \%$ para os casos com tuba leve e moderadamente acometida, respectivamente. Tais resultados corroboram a hipótese de que o grau de acometimento da tuba, em especial das fimbrias, relaciona-se diretamente à probabilidade de gestação intra-uterina ${ }^{2,8,9}$.

As taxas cumulativas de gravidez verificadas após um e dois anos $(25,0$ e 34,4\%, respectivamente) são semelhantes aos dados disponiveis na literatura internacional ${ }^{10-14}$. Taxas mais elevadas são descritas em estudos que incluem apenas fimbrioplastias simples ${ }^{15,16}$.

Relata-se incidência de gestação ectópica de 2 a $18 \%$ após microcirurgia tubária e 5 a $16 \%$ após laparoscopia ${ }^{11,17}$. Ocorreu apenas um caso de gestação ectópica na presente casuística $(9,1 \%$ de todas as gestações).

Atualmente, vários autores recomendam a 
FIV como primeira alternativa para todos os casais inférteis, devido à presença de lesão tubária, atingindo taxas de gestação de até $46 \%^{18-21}$. Entretanto, o custo elevado, o risco de gestações múltiplas e a necessidade de repetição da técnica se a paciente desejar mais de um filho são fatores que devem ser considerados antes da indicação de FIV.

Acreditamos que para pacientes jovens, sem a presença de nenhum outro fator associado que comprometa a fertilidade do casal, pode-se oferecer a correção cirúrgica como opção inicial de tratamento nos casos de hidrossalpinge leve e moderada. Preferimos a abordagem laparoscópica, pois é menos invasiva, com recuperação mais rápida e resultados semelhantes aos da microcirurgia em termos de taxa de gestação. Por outro lado, aquelas pacientes com prognóstico reservado devido à idade avançada, lesões tubárias graves ou presença de outros fatores associados têm como melhor opção técnicas de fertilização assistida.

\section{SUMMARY}

Purpose: to analyze the pregnancy rates after laparoscopic and microsurgical treatment of hydrosalpinx.

Methods: from July 1996 to May 1999 thirty-nine infertile patients with hydrosalpinx were treated according to a previously approved research protocol. They were randomly divided into two groups, according to the previously proposed surgical approach: laparoscopic or open microsurgical salpingostomy. To analyze the results, patients were stratified according to tubal damage, and pregnancy rates in both groups were determined for 24 months.

Results: pregnancy rates in our series were 35.3 and $33.3 \%$ after laparoscopic and microsurgical salpingostomy, respectively. According to the severity of tubal damage, patients with mildly and moderately damaged tubes got pregnant in 66.7 and $21.7 \%$ of the cases, respectively. Cumulative pregnancy rates in one and two years were 25.0 and $34.4 \%$, respectively. There was a single case of ectopic pregnancy, corresponding to $9.1 \%$ of all pregnancies.

Conclusion: patients with mild and moderate lesions may be initially treated with surgery, and conception success is inversely proportional to the degree of tubal damage.

KEY WORDS: Infertility. Hydrosalpinx. Laparoscopy. Microsurgery.

\section{Referências}

1. Gocial B. Primary therapy for tubal disease: surgery versus IVF. Int J Fertil Menopausal Stud 1995; 40:297-302.
2. Dubuisson JB, Chapron C, Morice F, et al. Laparoscopic salpingostomy: fertility results according to the tubal mucosal appearance. Hum Reprod 1994; 9:334-9.

3. McComb PF, Paleologou A. The intussusception salpingostomy technique for the therapy of distal oviductal occlusion at laparoscopy. Obstet Gynecol 1991; 78:443-7.

4. The American Fertility Society classifications of adnexal adhesions, distal tubal occlusion, tubal occlusion secondary to tubal ligation, tubal pregnancies, müllerian anomalies and intrauterine adhesions. Fertil Steril 1988; 49:944-55

5. Swolin K. 50 Fertilitätsoperationen. Acta Obstet Gynecol Scand 1967; 46:234-68.

6. Audebert AJ, Pouly JL, Von Theobald P. Laparoscopic fimbrioplasty: an evaluation of 35 cases. Hum Reprod 1998; 13:1496-9.

7. Broadbent M, Magos AL. In-vitro fertilisation or tubal surgery (letter). Lancet 1991; 337: 1291-2.

8. Winston RM, Margara RA. Microsurgical salpingostomy is not an obsolete procedure. $\mathrm{Br}$ J Obstet Gynaecol 1991; 98:637-42.

9. Marana R, Rizzi M, Muzii L, Catalano GF, Caruana P, Mancuso S. Correlation between the American Fertility Society classifications of adnexal adhesions and distal tubal occlusion, salpingoscopy, and reproductive outcome in tubal surgery. Fertil Steril 1995; 64:924-9.

10. Dubuisson JB, Bouquet de Joliniere J, Aubriot FX, Darai E, Foulot H, Mandelbrot L. Terminal tuboplasties by laparoscopy: 65 consecutive cases. Fertil Steril 1990; 54:401-3.

11. Dlugi AM, Reddy S, Saleh WA, Mersol-Barg MS, Jacobsen G. Pregnancy rates after operative endoscopic treatment of total (neosalpingostomy) or near total (salpingostomy) distal tubal occlusion. Fertil Steril 1994; 62:913-20.

12. Jacobs LA, Thie J, Patton PE, Williams TJ. Primary microsurgery for postinflammatory tubal infertility. Fertil Steril 1988; 50:855-9.

13. Singhal V, Li TC, Cooke ID. An analysis of factors influencing the outcome of 232 consecutive tubal microsurgery cases. Br J Obstet Gynaecol 1991; 98:628-36.

14. Taylor RC, Berkowitz J, McComb PF. Role of laparoscopic salpingostomy in the treatment of hydrosalpinx. Fertil Steril 2001; 75:594-600.

15. Patton GW Jr. Pregnancy outcome following microsurgical fimbrioplasty. Fertil Steril 1982; $37: 150-5$.

16. Milingos SD, Kallipolitis GK, Loutradis DC, et al. Laparoscopic treatment of hydrosalpinx: factors affecting pregnancy rate. J Am Assoc Gynecol Laparosc 2000; 7:355-61. 
17. Penzias AS, DeCherney AH. Is there ever a role for tubal surgery? Am J Obstet Gynecol 1996; 174:1218-23.

18. Watson A, Vandekerckhove P, Lilford R. Techniques for pelvic surgery in subfertility. Cochrane Database Syst Rev 2000; 2:CD000221.

19. Holst N, Maltau JM, Forsdahl F, Hansen LJ. Handling of tubal infertility after introduction of in vitro fertilization: changes and consequences. Fertil Steril 1991; 55:140-3.
20. Chardon C, Boulieu D, Rochet Y, Payan F, Ayzac L. Stérilité d'origine tubaire: quel traitement proposer: FIV ou chirurgie? Rev Fr Gynécol Obstét 1992; 87:355-60.

21. Aboulghar MA, Mansour RT, Serour GI. Controversies in the modern management of hydrosalpinx. Hum Reprod Update 1998; 4:88290.

\section{Congresso Brasileiro de Obstetrícia e Ginecologia da Infância e Adolescência}

\section{6 a 19 de outubro de 2002}

\section{Cuiabá - MT}

\section{Informações: \\ (0XX65) 623-7233}

